Themenheft Nr. 44: Datengetriebene Schule.

Forschungsperspektiven im Anschluss an den 27. Kongress der DGfE

Herausgegeben von Mandy Schiefner-Rohs, Sandra Hofhues und Andreas Breiter

\title{
¿... dass man denen auch mal 'nen Spiegel vorhalten kann)
}

\author{
Metaphern im Diskurs um Daten (in) der Schule \\ Michael Becker ${ }^{1}$ (D), Ulrike Krein ${ }^{1}$ (D) und Mandy Schiefner-Rohs ${ }^{1}$ (D) \\ ${ }^{1}$ Technische Universität Kaiserslautern
}

\begin{abstract}
Zusammenfassung
Die Erzeugung und Nutzung (digitaler) Daten gewinnt nicht nur im unterrichtlichen Kontext zunehmend an Relevanz, sondern Daten beeinflussen auch das Handeln pädagogischer Akteurinnen und Akteure und stellen diese vor (neue) Herausforderungen. Der Beitrag fokussiert hieran anknüpfend das Verständnis von Datenhandeln pädagogischen Personals in dessen Alltag. Unter der Prämisse, dass Metaphern Denk- und Handlungsorientierungen sichtbar und damit auch Un-Sagbares sowie handlungsleitende Sinnstrukturen zum Ausdruck bringen können, rekonstruieren wir die in diesem Zusammenhang gebrauchte Verwendung metaphorischer Konzepte in Interviews mit Schulleitungen. Nach einer kurzen theoretischen und methodischen Rahmung des Artikels werden im empirischen Ergebnisteil vier rekonstruierte metaphorische Konzepte (Datenhandeln ist verwalten, Datenhandeln ist schützen, Datenhandeln ist sichtbarmachen sowie Datenhandeln ist Waren austauschen) vorgestellt und diskutiert. Diese unterscheiden sich zwar hinsichtlich ihrer Funktionen, weisen aber als Common Sense auf ein weitestgehend vergegenständlichtes Verständnis von Daten in Schule hin.
\end{abstract}

(... to hold a mirror up to them sometimes'. Metaphors in the Discourse about Data (in) School

\begin{abstract}
The production and use of (digital) data is not only becoming increasingly relevant in the school context, but also has a concrete impact on the actions of pedagogues and poses (new) challenges for them. Following on from this, the article focuses on the understanding of data practices of school leaders in their everyday work. Under the premise that metaphors can make orientations of thought and action visible and thus express the unsayable, we reconstruct the use of metaphorical concepts in interviews with school leaders. After a brief theoretical and methodological framing of the article, the empirical
\end{abstract}


results section presents and discusses the four metaphorical concepts (data practices are administration, data practices are protection, data practices are visualization, and data practices are exchange of goods). The metaphorical concepts of data action in schools differ in terms of their functions, but as common sense they point to a largely passive understanding of data in schools.

\section{Datafizierung (in) der Schule als aktuelles Phänomen}

Der Umgang mit Daten ist per se nichts Neues für Pädagogik und Schule. Daten nehmen schon seit einigen Jahren Einfluss auf schulische Praxis, wie die Diskussionen um Educational Governance und damit verbundene schulinterne oder externe Evaluationen deutlich machen (vgl. z. B. Altrichter und Maag Merki 2010; van Ackeren 2003; van Ackeren, Klemm, und Kühn 2015). In einer Kultur der Digitalität (Stalder 2016) gibt es allerdings Verschiebungen dieser allgemeinen Entwicklung des zunehmend datenbasierten Handelns in der Schule: Erstens ist es einfacher geworden, Daten zu erzeugen und zu nutzen. Zweitens können vielfältige Bereiche und Handlungsfelder von Schule durch (digitale) Daten erschlossen werden. Drittens können Daten zusammengeführt und somit auch erweiterte Aussagen z. B. über Lernleistungen oder Verhalten getroffen werden. Daten scheinen hierbei «stets für etwas gegeben zu sein [...]: für ihre spätere Interpretation, für ihre computertechnische Auswertung, für den späteren Zugriff und für das Verständnis von Wirklichkeit» (Gießmann und Burkhardt 2014, 3).

Das Soziale zeigt sich so «in steigendem Maße datenvermittelt und datenbasiert, wenn nicht gar datengetrieben» (Houben und Prietl 2018, 7) und die Erzeugung, Erhebung, Speicherung und Auswertung von Daten aller Art wird (schul-)alltagsprägend. Dieser unter Datafizierung gefasste verdichtete, komplexe Prozess der Kommunikation und Interdependenzbewältigung zwischen Akteurinnen und Akteuren und ihren Handlungskontexten qua Daten (Breiter und Jarke 2019) hat Implikationen für die Gestaltung von Schule: Die mit einer Datafizierung der Gesellschaft einhergehende Expansion von Datenerhebungen und -verarbeitungsformen in Form von (pädagogischer) Steuerung durch Zahlen (Grek 2009; Hartong 2016) oder die Quantifizierung des Sozialen (Mau 2017) schliesst ebenfalls die Optimierung des Lehrens und Lernens und darauf fokussierte Praktiken ein. So werden Daten nicht mehr nur zweckgerichtet (beispielsweise für Evaluationen) generiert, auch können sie in Form von digital traces, die beispielsweise in Schulinformationssoftware hinterlassen werden (Breiter und Hepp 2018), als Datenquellen erschlossen werden. Nicht selten werden so auch Anreizstrukturen zur Datenerzeugung vielfältigster Art geschaffen. Digitale Daten werden zu (scheinbar objektiven) Referenzpunkten für individuelle oder gemeinsame, implizite oder explizite (pädagogische) Entscheidungen und haben Einfluss auf schulisches Handeln. Verbunden mit solchen Optimierungstendenzen sind 
Bestrebungen hinsichtlich der Individualisierung von Lehr-Lernprozessen, welche häufig gemeinsam mit Automatisierung und Adaptivität als Potenzial der Nutzung digitaler Daten proklamiert werden (beispielsweise Dander und Aßmann 2015). Verkannt wird, dass Daten nicht nur soziale Wirklichkeit beschreiben, sondern diese auch erschaffen bzw. verändern. Software bzw. Dateninfrastrukturen sind soziale Relationen technisch inhärent, wodurch diese nicht als neutral angenommen werden können (Dalton und Thatcher 2014; Fuller 2008; Kitchin und Lauriault 2014; Lachney, Babbitt, und Eglash 2016).

Während solche Optimierungstendenzen und Individualisierungsansprüche im Hochschulkontext vor allem unter dem Begriff Learning Analytics bereits (auch kontrovers) verhandelt werden (z. B. Ifenthaler und Schumacher 2016; Seiler et al. 2018), zeigen sich Praktiken und Diskussionen darüber an und in Schule zögerlicher bzw. weniger verbreitet (Selwyn 2020). Zu fragen ist daher, wo und wie damit zusammenhängende Perspektiven in Schule eigentlich verhandelt werden. Zudem stellen datengestützte Entscheidungen Pädagoginnen und Pädagogen vor die Anforderung, sich selbst zu Prozessen der Datafizierung zu positionieren. Diese Positionierungen werden hier in den Blick genommen. Denn um sich aber positionieren zu können, braucht es ein Verständnis davon, was Datafizierung bzw. Daten und das Handeln mit innen bedeuten und wie dieses gerahmt wird. Bezogen auf digitale Daten und den Prozess der Datafizierung, so die Überlegung des Beitrags, ist dieses Verständnis aber nicht so eindeutig. Diese Fragen werden im Folgenden mit Bezug auf die Schule fokussiert: Welche Bedeutung haben Daten im Alltag von Schulleitungen? Wie wird über Datenhandeln geredet und welche Perspektiven auf Datafizierung werden darin sichtbar?

\section{Metaphern als (erster) Zugang zu Datenpraktiken (in) der Schule}

Um diesen Perspektiven auf Daten in der Schule und den oben skizzierten Fragen nachzugehen, soll in einem ersten Schritt eine Analyse erfolgen, wie über Daten und ihre Erzeugung an Schule gesprochen wird. In diesen Versprachlichungen liegt eine Perspektive von Weltwahrnehmung, die auf mögliche, sich in Schule zeigende Praktiken hinweist. Daher erscheint es zielführend, die Metaphern, welche in der Schule im Zusammenhang mit der Gestaltung pädagogischer Wirklichkeit durch und mit (digitalen) Daten genutzt werden, genauer zu analysieren. Metaphern können dabei als Spiegel der persönlichen Realität bzw. als Sinn herstellendes Werkzeug (Saban 2004) betrachtet werden. Damit leisten sie die

"Strukturierung einer noch unbegriffenen Erfahrung mit Hilfe der Begrifflichkeit aus einem vertrauten Erfahrungsbereich. [...] Metaphern helfen uns, Wirklichkeiten zu erschließen, Erfahrungen zu artikulieren und Neues zu denken» (Herzog 1994, 2). 
Gerade der letzte Aspekt ist bezogen auf Datenhandeln in der Schule von besonderem Interesse. Datenhandeln begreifen wir daher als diskursive Praktik, aus welcher Subjektpositionen entlang spezifischer «Regeln, Differenzlinien und Grenzen des Erkennbaren» (Dander 2015, 13) hervorgebracht werden respektive sich auf Ebene von Institutionen als relative Verfestigungen diskursiver Strukturierungen zeigen (ebd.). Artikelleitend sind somit Fragen nach der Gestaltung von Schule unter Perspektive digitaler Daten sowie der Eröffnung von Bildfeldern mit gewählten Metaphern im schulischen Diskurs. Diese können Aufschluss darüber geben, welche Strukturen in den konkreten Praktiken aufgeführt werden und welche Elemente auf diese Weise auch verschwinden.

Rekurrierend auf die kognitive Metapherntheorie nach Lakoff und Johnson (1980) wurde mit der systematischen Metaphernanalyse (Schmitt 2017; Schmitt, Schröder, und Pfaller 2018) ein hermeneutischer, sinnrekonstruierender methodischer Ansatz (ebd.) für die vorliegende Studie gewählt. Ziel ist es, metaphorische Konzeptualisierungen zu rekonstruieren, sie in ihrer Beziehung zueinander zu analysieren sowie die damit denkleitende Wirkung im Diskurs um Datafizierung (in) der Schule zu reflektieren.

Metaphorische Konzepte werden hierbei begriffen als Bündelung mehrerer metaphorischer Redewendungen im Sinne spezifischer «individuelle[r] oder kulturelle[r] Muster des Denkens, der Wahrnehmung, der Empfindung und des Handelns» (Schmitt, Schröder, und Pfaller 2018, 25), in welchen sich Sinnstrukturen und soziale Orientierungsmuster dokumentieren. Anknüpfend an das Verständnis von Metaphern nach Lakoff und Johnson (1980) $)^{1}$ wird im vorliegenden Beitrag von metaphorischen Redewendungen respektive Metaphern gesprochen, wenn ein Wort bzw. eine Wendung verwendet wird, welche neben der wörtlichen Bedeutung, die einem prägnanten Bedeutungsbereich entstammt, eine weitere Bedeutung für den relevanten Kontext der Sprachäusserung aufweist, die zeitgleich auf einen zweiten, abstrakten Bereich übertragen wird. Der prägnante Bedeutungsbereich, dem die wörtliche Bedeutung der Metapher entstammt, wird als Quellbereich bezeichnet, der den Erfahrungsraum abbildet, aus welchem sich das Wort bzw. die Wendung speist (Schmitt, Schröder, und Pfaller 2018). Die Übertragung der wörtlichen Bedeutung geschieht auf den sogenannten Zielbereich, welcher beschreibt, worüber gesprochen wird. Die Übertragung von Quell- auf Zielbereich dient hierbei «der konstruierenden Versprachlichung des Zielphänomens ebenso wie seiner sozialen Rezeption» (ebd., 3).

Innerhalb der sozialwissenschaftlichen Metaphernanalyse ist es von Bedeutung, dass sich solche metaphorischen Übertragungen nicht rein individuell gestalten, sondern auf kulturellen Denkmustern fussen und diese selektiv und partiell darbieten

1 Der Begriff der Metapher ist ein umstrittenes und abstraktes Konstrukt, dessen eindeutige definitorische Bestimmung in den aktuellen theoretischen Diskursen bislang aussteht (detaillierte Ausführung siehe Schmitt, Schröder, und Pfaller 2018). 
(Lakoff und Johnson 1980), was eine Rekonstruktion und Reflexion dieser ermöglicht. Schmitt und Kolleginnen (2018) weisen darauf hin, dass diese weitgefasste Definition des Metaphernbegriffs für die Identifikation von Metaphern auch der Berücksichtigung von Grenzphänomenen wie z. B. «toten» Metaphern², Metonymien³, kausalen und finalen Konjunktionen, Adverbien und Präpositionen oder Vergleichen als Metaphern bedarf ${ }^{4}$, welche im Rahmen der vorliegenden Studie berücksichtigt wurden.

\subsection{Forschungsdesign und Stichprobe}

Der Studie liegt ein qualitatives Forschungsdesign zugrunde, in dem mittels Fallstudien metaphorische Konzepte spezifischer Gruppen (Schmitt, Schröder, und Pfaller 2018, 96) rekonstruiert werden. Hierfür wurden leitfadengestützte Interviews mit einer Dauer von ca. 15 bis 45 Minuten zum Thema «Datafizierung (in) der Schule» geführt und in die Analyse einbezogen. Die Interviews entstanden in der Sensibilisierungsphase eines Projekts ${ }^{5}$, um den Kontext von Schule und Daten ${ }^{6}$ näher in den Blick zu nehmen. Interviewt wurden insgesamt fünf rheinland-pfälzische Schulleitungen ${ }^{7}$. Fokussiert wurde, wie Schulleitungen und Lehrkräfte in der Schule über digitale Daten sprechen, diese verstehen und mit ihnen umgehen.

Das Vorgehen der Metaphernanalyse gestaltete sich in Anlehnung an Schmitt (2017) zweigeteilt: Nach der Definition des Zielbereichs «Daten(handeln) (in) der Schule» wurden (1) zunächst möglichst alle Metaphern mit Bezug zu eben diesem Zielbereich identifiziert und gesammelt, bevor diese (2) für die Rekonstruktion metaphorischer Konzepte herangezogen wurden. Die einzelnen Metaphern werden so unter den jeweiligen Konzepten verdichtet.

2 Tote Metaphern sind Metaphern, deren Bedeutungen in den allgemeinen Sprachgebrauch übergegangen sind, so dass es dafür kein anderes Wort mehr gibt, z. B. Buchrücken oder Tischbein.

3 Das Stilmittel der Übertragung bzw. Subsumtion, z. B. «Gehen wir ein Glas trinken.»

4 Für eine ausführliche Darstellung dieser Grenzphänomene siehe Schmitt, Schröder und Pfaller 2018, 99-104.

5 Die Interviews dienten der Vorbereitung auf das BMBF-Projekt «All is data - die (gem)einsame Suche nach Erkenntnis in einer digitalisierten Datenwelt» mit den Förderkennzeichen 01JD1903. https://all-is-data.de

6 Die Interviewten geben ihr eigenes Datenverständnis wieder; ihnen wurde kein Datenverständnis vorgegeben (dazu Kapitel 4).

7 Drei Interviews wurden vor der Ausbreitung von Covid-19 zu Beginn 2020 geführt, die übrigen unmittelbar im Anschluss an die coronabedingten Schulschliessungen in Deutschland. Diese dienten einer ersten Exploration der Relevanz und Nutzung (digitaler) Daten in Schule, wodurch sich die Interviewfragen respektive Erzählimpulse, gerahmt durch die steigende gesellschaftliche Relevanz datengestützter Entscheidungen, auch auf die Rolle und Nutzung von Daten in Schule bezogen. 


\section{Ergebnisdarstellung}

Im Rahmen der Interviewanalyse zeigte sich eine hohe inter- und intrapersonale Metaphernpluralität; einerseits hinsichtlich der Differenz der gewählten Metaphorik, andererseits auf quantitativer Ebene hinsichtlich der Metaphernvielfalt. Unabhängig von metaphorischen Konzepten, die nur innerhalb einzelner Interviews gesättigt wurden $^{8}$ und damit auf individuelle metaphorische Konzepte hinweisen, konnten über alle einbezogenen Interviews hinweg und damit als kollektive bzw. als Indiz auf geteilte Sinnkonstruktionen vier metaphorische Konzepte rekonstruiert werden:

1. Datenhandeln ist verwalten.

2. Datenhandeln ist schützen.

3. Datenhandeln ist sichtbarmachen.

4. Datenhandeln ist Waren austauschen.

Im Folgenden werden wir diese metaphorischen Konzepte mit den dazugehörigen Metaphern vorstellen, interpretieren und eine Diskussion mit Blick auf Implikationen für Datenhandeln in der Schule anschliessen.

\subsection{Datenhandeln ist verwalten}

Die dominantesten metaphorischen Wendungen der Untersuchung können unter dem metaphorischen Konzept Datenhandeln ist verwalten subsumiert werden. Datenhandeln wird hierbei von den Schulleitungen mit verwaltungsgenuinen Aufgaben wie der Dokumentation, Strukturierung, Anmeldung, Erfassung, Entlassung und Speicherung von Daten verhandelt.

"Schüler werden aufgenommen, Schüler werden entlassen. Ja, das ist wohl das grundlegende an Schulen. Ich melde mich an, und sie melden ihre Tochter ihre Söhne, keine Ahnung ihrer Enkel, die melden sie an.» (SL1, Pos. 3, Herv. Autoren)

"Sind ja die Daten, die wir dann als Schule erzeugen. Einmal nehme auf, dann wird er entlassen, und zwischendrin gibt es irgendwelche Noten, die erfasst werden.» (SL1, Pos. 7, Herv. Autoren)

Schulleitungen sowie Lehrende werden im Datenhandeln dazu angehalten, Daten zu erfassen und zu kontrollieren, dass bzw. ob entsprechende Vorgaben eingehalten werden und Prozesse bestimmungsgemäss ablaufen. Datenhandeln konstituiert sich folglich auch als bürokratischer Akt der Kontrolle über die Rechtmässigkeit des Umgangs mit Daten. Die Relevanz und Unanfechtbarkeit von Datenschutzrichtlinien

8 Durch die Artikellänge können nur interviewübergreifend rekonstruierte metaphorische Konzepte aufgenommen werden. Zur Kontrastierung werden in Kapitel 4 weitere Metaphern angeführt. 
zeichnet sich interviewübergreifend bei den Schulleitungen ab und wird z. B. im Rahmen von Arbeitsprozessen und Handlungsweisen betont:

«Entweder ist da haben Sie da was weiß ich fünf Firewalls drauf oder das Passwort nur was weiß ich von der Sekretärin, die dann auf alles draufschauen kann. Und bei diesem neuen Programm ist gewährleistet, dass alle Daten nach drei Jahren auch gelöscht werden. Also da wird auch nach den EU Datenschutzrichtlinien entsprechend gearbeitet.»(SL1, Pos. 5, Herv. Autoren)

Wird nach der Funktion des Verwaltens als metaphorisches Konzept gefragt, ist anzumerken, dass die Schulleitungen Datenhandeln vor allem unter den Aspekten Macht, Bürokratie und Kontrolle thematisieren. Das (individuelle wie organisatorische) Datenhandeln verfolgt somit die Intention einer kontrollgeleiteten Verwaltung von Schule. So wird die im etymologischen Ursprung des Wortes «verwalten» (als Zuständigkeit respektive Administration von Daten) inhärente Machtstruktur auch in den Interviews deutlich, wenn dort schulisches Personal vor allem als aktiv handelnd beschrieben wird, dem die Dokumentation, Ablage und Nutzung von Daten obliegt und somit auch Macht hat, über diese zu verfügen. Das verbalisierte Datenhandeln basiert, abseits der individuellen Macht der Agierenden über Daten, auf gesetzlichen Vorgaben durch die Datenschutzgrundverordnung (DSGVO) als normgebende Ins$\operatorname{tanz}$.

Im Rahmen eines solchen Verständnisses von Datenhandeln (als Form von Kontrolle, Macht und Bürokratie) werden Aspekte wie Innovation und Zukunftsorientierung 9 aussen vor gelassen. So wird Datenhandeln von den Schulleitungen im Rahmen dieses Konzeptes lediglich als verwaltungsorientierte Aufgaben begriffen, die jedoch nicht über das Aufnehmen und Hinterlegen von Daten hinausgeht:

«Ja, aber wir selbst haben jetzt mit der Statistik, [...], nix zu tun, [...] wenn macht das überhaupt eine Übergeordnete Instanz, die Zugriff auf unsere Daten wiederum hat aber das betrifft uns als Schulleitungen und auch als Lehrer eigentlich wenig.» (SL3, Pos. 32, Herv. Autoren)

Es ist darauf hinzuweisen, dass die untersuchten Interviews mit Schulleitungen geführt wurden, welche qua Aufgabenvielfalt u.a. mit der Führung und dem Management von Schule und somit auch mit Verwaltungsaufgaben betraut sind. Die hier identifizierte Dominanz dieses metaphorischen Konzepts ist demnach unter der Restriktion der Gruppe der Befragten und dem Einfluss ihrer alltäglichen Aufgaben zu reflektieren und erfordert zukünftig Folgeuntersuchungen mit weiteren schulischen Beteiligten ${ }^{10}$.

9 Dies steht beispielsweise im Kontrast zu metaphorischen Wendungen des Glaubens (Vision; wunderbare Sachen machen), welche innerhalb eines der untersuchten Interviews identifiziert werden konnten und das Potential von Datenhandeln für Innovationen in der Gestaltung von Lehr-/Lernprozessen betonen.

10 Dies gilt für alle hier vorgestellten metaphorischen Konzepte, jedoch in besonderem Masse für das Konzept Datenhandeln ist verwalten. 


\subsection{Datenhandeln ist schützen}

Anknüpfend an die in der Pädagogik verbreiteten Metaphern der Schule als Garten (vgl. Guski 2007) und somit als Schonraum, in dessen Abgeschlossen- und Geschütztheit es «die freie Entfaltung vor schädlichen äußeren Einflüssen zu bewahren gilt» (Guski 2007, 169), lassen sich ähnliche metaphorische Wendungen in den von uns analysierten Interviews finden: Im Datenhandeln und dem damit unweigerlich verknüpften und mehrfach betonten Anspruch eines Datenschutzes wird ein geschützter Umgang interviewübergreifend akzentuiert. Der hier angesprochene Umgang konstruiert sich als eine empfindsame und feinfühlige (intim; ordentlich), vielleicht auch durch Verordnungen besonders relevante Beschäftigung mit Daten, deren Besonderheit und Relevanz explizit hervorgehoben wird:

"Und also wir erfahren da sehr intime Dinge eigentlich und da muss man auch sehr geschützt mit umgehen. Und das ist eine wichtige Angelegenheit. Also, dass wir hier sensibel damit umgehen. Das ist für mich ein absolutes Muss.» (SL4, Pos. 2, Herv. Autoren)

«Der Lehrer unterschreibt auch, dass er die Datenschutzverordnung gelesen hat, dass er ordentlich mit seinen Daten umgeht. Also wird jeder selbst an seiner Verpflichtung genommen, auch wenn es nur eine Vertretungskraft da ist, die sechs Wochen bei uns unterrichtet.» (SL1, Pos. 35, Herv. Autoren)

Betont das erste Zitat vornehmlich die Notwendigkeit eines sensiblen und geschützten Umgangs, rücken im zweiten Beispiel, analog zum Konzept des Verwaltens, auch Aspekte der Verantwortung des Einzelnen und der damit verbundenen Gesetzgebung in den Vordergrund. Interessant ist der Verweis auf die individuelle Ebene und Verantwortung: Es handelt «jeder halt nach Vorgabe des Landes mit mehr oder weniger guten oder schlechten Gefühl» (SL3, Pos. 30) und somit letztlich stark geprägt durch (eigene) Ermessensentscheidungen. Vor dem Hintergrund, dass die einzelnen Akteurinnen und Akteure zur Verpflichtung herangezogen werden, was neben einer grossen (individuellen) Verantwortung gleichermassen eine Verbindlichkeit des Tuns evoziert, erhält der Aspekt der Verantwortung im metaphorischen Konzept des Schützens eine besondere Perspektive.

«Also ich merke das an mir wo ich verantwortlich dafür bin, dass ich sehr viel mehr an Detailarbeit machen muss und auch nicht immer erkennen kann, wenn ich an einer Stelle etwas tue, welche Konsequenzen hat das andere im anderen Programm?» (SL4, Pos. 12, Herv. Autoren)

Zeigt sich beispielhaft anhand dieses Zitats nicht nur das Bewusstsein bzgl. der Verantwortung, wird hierbei gleichermassen auf ein damit einhergehendes erhöhtes Arbeitsaufkommen hingewiesen, welches eine «große Sorgfalt [erfordert]» (SL4, Pos. 
12) und mit nicht absehbaren Konsequenzen einhergeht. Gleichermassen ist das hier beschriebene von hoher Verantwortung geprägte individuelle Datenhandeln parallel zum Konzept des Verwaltens durch die DSGVO sowie Landesvorgaben in einen (kollektiven) gesetzlichen Rahmen für einen normgerechten Umgang mit Daten eingebettet.

Doch lassen sich nicht nur entlang der gesetzlichen Vorgaben Parallelen zum auf den ersten Blick gegensätzlichen metaphorischen Konzept Datenhandeln ist verwalten identifizieren: Sind dem vorangegangenen Konzept Machtstrukturen qua Etymologie inhärent, so können auch bei diesem metaphorischen Konzept Machtperspektiven identifiziert werden. Erscheinen Daten im metaphorischen Konzept des Schützens als Schutzbedürftige und somit als Entitäten, welche des Schutzes anderer bedürfen oder mit anderen Worten auf deren Betreuung respektive Schutz angewiesen sind, wird hier ein hierarchisches Verhältnis zwischen Daten und den Beteiligten deutlich: Schulische Akteurinnen und Akteure stehen schützend über den Daten und sehen sich damit (auch) in einer Machtstellung, diese vor möglichen Gefahren zu bewahren. Das Konzept Datenhandeln ist schützen gestaltet sich demnach am Beispiel der hier analysierten Interviews als eine mit Verantwortung einhergehende Beschäftigung der Akteurinnen und Akteure mit Daten, welche wiederum personifiziert als schutzbedürftig adressiert werden. Wenn also im pädagogischen Bereich Schutzbefohlene Kinder (und Jugendliche) waren, geht der Schutz auf ihre Daten über. Ob Daten nur pars pro toto für Schülerinnen und Schüler stehen oder diese hier zu Datenpunkten werden, kann nicht abschliessend geklärt werden, gleichwohl werden Schülerinnen und Schüler in der Wahrnehmung von Lehrkräften so auf ihre Daten reduziert.

Eingedenk, dass Schutz beispielsweise in Form von Verteidigung, Aufsicht oder Ein- bzw. Abgrenzung geschehen kann, ist auffallend, dass keine metaphorischen Konzepte gefunden werden konnten, die auf eine solche Bedeutung hinweisen. Gleichermassen verbleiben der oben skizzierten Denkstruktur folgend technische Aspekte, der Datenbeschaffenheit per se aber auch der Umgang mit diesen, ungeachtet. Weitere ungeklärte Aspekte innerhalb der Schulleitungsinterviews sind die Intention der Schutzhandlung sowie mögliche Gefahren, vor denen es Daten zu schützen gilt.

\subsection{Datenhandeln ist sichtbarmachen}

Das weitere, aus den Interviews rekonstruierte metaphorische Konzept ist eng verwandt mit dem in der Pädagogik relevanten Konzept des Sehens und Sichtbarmachens. Metaphern des Sehens weisen auf eine lange Tradition für den gesamten Bereich der Erkenntnis hin (u. a. Gering 2007). Metaphorische Wendungen in unseren Interviews lassen sich in zwei unterschiedlichen Perspektiven fassen: Zum einen die Sichtbarmachung als Teil einer (wissenschaftlichen) Auseinandersetzung, zum 
anderen als Teil des Sehens. Dominante Motive innerhalb dieses Konzepts sind Auswertung, Evaluation, Erheben, Statistik, (Stärken und Schwächen) Erkennen, Häufigkeiten, in den Blick nehmen, Reflexion oder Spiegel. Gerade die Spiegel-Metapher wird zentral mit der Perspektive des Datenhandelns verbunden: So geht es einerseits darum, dass man mittels Daten Schülerinnen und Schüler den Spiegel vorhalten, also somit Erziehungsprozesse sichtbar- und legitimierbar machen kann.

"Letzten Endes auch wirklich so im Sinn auf diese Kontrolle die man auch hat um sicherzustellen das halt gerade Schüler, die im Notenheft net so gut dastehen und dann immer wieder vorgeben: Aber ich lerne ja die Vokabeln das man denen auch mal 'nen Spiegel vorhalten kann. Und denen sagen kann ja so über diese App sehe ich halt du warst jetzt in der Woche nur 1-2 mal eingeloggt deine Mitschüler gleich die waren jeden Tag 10 Minuten aktiv und das wir da dabei einfach quasi den Spiegel vorhalten kann und sagen kann, auf Grund dieser Lage wer kann gibt es keinen Nachteilsausgleich wie auch immer.» (SL3, Pos. 12, Herv. Autoren)

Andererseits kommt der Spiegel auch als Feedback- bzw. Reflexionsinstrument zur Sprache, indem Daten dazu genutzt werden, Erkenntnisse zu gewinnen, um das eigene Handeln im wahrsten Sinne des Wortes «in den Blick zu nehmen»:

«Ja, ich habe aber nicht die Wahrnehmung, dass diese Daten an anderer Stelle in irgendeiner Form denn auch mal uns widergespiegelt werden.» (SL1, Pos. 52, Herv. Autoren)

«Das nutzen die noch nicht also da gibt es Ideen, Ansätze sowas zu machen, auch vor dem Hintergrund, dass man, das andere Schulen schon mal überlegt haben wie kann man beispielsweise die Abschlussquote erhöhen, dass man da mal draufschauen Mensch, wie lange das jetzt jemand mit drin? Wie haben sich die Noten entwickelt, dass wir darüber mal Statistiken führen, sowas, wird bei uns da net umgesetzt» (SL1, Pos. 41, Herv. Autoren)

Innerhalb des Konzepts des Sichtbarmachens können verschiedene Ebenen unterschieden werden: So erreichen Daten durch die Sichtbarkeit einen gewissen Wahrheitscharakter, der im weiteren Prozess nicht mehr angezweifelt oder hinterfragt wird. Durch das Sichtbarmachen wird soziale Wirklichkeit geschaffen, die dann quasi unhinterfragt für sich steht. Die Perspektive, dass Daten auch Ausdruck vielfältiger Setzungen und damit (auch) subjektiv sind, wird an keiner Stelle innerhalb der Interviews reflektiert. Damit einher geht eine gewisse Verantwortungsübergabe an Daten, die kaum bis gar nicht hinterfragt wird: Wenn es Daten gibt, dann sind diese vermeintlich objektiv, können also von jedem gesehen werden. Zudem werden Daten unter dem Aspekt der Sichtbarmachung genutzt, um Entscheidungen zu legitimieren 
oder Daten in ihrer Herstellung und Offenlegung von per se unsichtbaren Prozessen und Konstrukten (Qualität, Lernen, gute Schule) zur Gestaltung von sozialen Prozessen an Schule heranzuziehen.

«Darüber wird auch gemessen dann, wie viele Abbrecher haben wir über das System? Wie viele laufen erfolgreich durch?»(SL1, Pos. 52, Herv. Autoren)

«Individualisiertes Lernen wirklich mit einer guten Software, die pädagogisch, die tatsächlich in der Lage ist, Dinge zu erkennen beim Schüler, Schwächen erkennen, Stärken erkennen kann und dann aber auch eine Auswahlmöglichkeit hat für Fördermöglichkeiten. Dass das eine Software auch wirklich leisten kann.»(SL4, Pos. 42, Herv. Autoren)

Das Sichtbarmachen als metaphorisches Konzept weist darauf hin, dass Daten in der Schule dazu genutzt werden, etwas, was sich ggf. nicht auf den ersten Blick erschliesst, in den Fokus zu nehmen und damit auch bearbeitbar zu machen. Dies können sowohl Aspekte der Auswertung, Evaluation und Statistik sein, indem mit Hilfe von Daten auch Qualität schulischer Prozesse sichtbar gemacht wird, als auch quantifiziert werden soll. Sichtbarmachen kann aber auch nach innen gerichtet werden, indem man Daten nutzt, um - im wahrsten Sinne des Wortes - in den Spiegel zu schauen und Reflexion anzuregen.

Die Auseinandersetzung mit der eigentlichen «Unsichtbarkeit» pädagogischer Praktiken und Prozesse ist per se nichts Neues (u.a. Treml 2000; Kade und Seitter 2007). So ist das Sichtbarmachen als metaphorisches Konzept eng mit bildhaften Diskursen in der Pädagogik verbunden (Pöggeler 1992). All diesen ist gemeinsam, dass sie das Innenleben von Bildungsprozessen in möglichst prägnanten Bildern zu veranschaulichen suchen, obgleich sich Bildung solchen bildgebenden Verfahren systematisch entzieht (vgl. auch Koselleck 2006, 105ff.). Hierzu werden nun Daten erzeugt und gewonnen, sei es auf Ebene der Schülerinnen und Schüler oder auf Ebene der Organisation Schule. Damit reiht sich das Konzept des Datenhandelns als Sichtbarmachen in genuin pädagogische Fragestellungen rund um die systematische Organisation von Lehr-Lernprozessen ein.

Bei der Funktion von Sichtbarmachen als metaphorisches Konzept rund um Datenhandeln fällt auf, dass Letzteres als Sichtbarmachung vor allem unter legitimatorischer Perspektive verhandelt wird, weiter sich hier Veränderungen in Bildungsinstitutionen zeigen, die unter dem Aspekt einer Öffnung von Schule diskutiert werden. War Lehren in früheren Zeiten meist ein recht «einsames Geschäft» von Lehrpersonen hinter verschlossenen Türen und die Gestaltung von Schule Aufgabe von Schulleitungen, mehren sich - nicht nur unter dem Blickwinkel von Qualitätssicherungsprozessen - die Rufe nach einer Öffnung der Lehre und Schule nach aussen (u. a. Forell, Bellenberg, und im Brahm 2019). Damit werden durch Datenhandeln 
unterschiedliche Funktionen wahrgenommen: Es wird der Dialog über Schule und/ oder Lernen sowohl nach innen als auch nach aussen angeregt, wodurch Qualitätsfragen adressiert werden können. Mit Sichtbarkeit verbunden sind aber auch Perspektiven von Legitimation, da diese Daten zur Rechtfertigung pädagogischen Handelns, z. B. gegenüber Eltern genutzt werden können. Datenhandeln wird so eine praktische Realisierung, um die vielschichtigen und kontingenten Handlungsräume in Lehr-Lern-Prozessen zu rekonstruieren.

Aber das metaphorische Konzept des Sehens kann nicht rekonstruiert werden, wenn nicht auch die darin liegenden blinden Flecken diskutiert werden, da die scheinbare Eingängigkeit des metaphorischen Konzepts von Sichtbarmachen auch einige Aspekte im Unklaren lässt. Denn Bildung und Lernprozesse sind vielschichtig und können beispielsweise kaum über Log-In Daten, Notendurchschnitte oder durch das Überwachen allein abgebildet werden (Krieter 2020; Krieter und Breiter 2020). Darüber hinaus bleibt in den Interviews unklar, was genau überhaupt mit Daten sichtbar gemacht wird bzw. gemacht werden kann. Am Beispiel von Yeats: «How can we know the dancer from the dance?» (Yeats 1949, 108) lässt sich die dahinterliegende epistemologische Schwierigkeit der «Sichtbarmachung» verdeutlichen (vgl. Schiefner und Eugster 2010):

«Auch wenn man fasziniert die Tanzenden in ihrer choreographierten Beweglichkeit beobachtet - dass man dabei den Tanz an sich wahrnimmt, ist so offensichtlich nicht. Um den Tanz zu erkennen, braucht es eine Abstraktionsleistung, die über die Beobachtung der Tanzhandlung hinausgeht und sich zugleich in einem Widerspruch verheddert. So ist der Tanz, was wir nicht sehen, wenn wir das Tun der Tanzenden als Tanz sehen. Es ist die alte hartnäckige Zirkularität des Wechselspiels von Form und Inhalt (Materie), die das verbindende Gemeinsame (die Form) von konkreten Handlungen (als der materiellen Grundlage eines Handlungsmusters) doch wieder nur im Rückbezug auf das einzelne Handeln eines einzelnen Handlungssubjekts begründen kann.» (ebd., 73)

Solches kann als Verweis auf die «unerwartete Unsichtbarkeit des wie selbstverständlich Sichtbaren» (ebd.) oder auf das generelle Problem der Operationalisierung gelten. So ist Sichtbarkeit im Datenhandeln eine Metapher, die uns im Umgang mit diesem Widerspruch hilft, dafür, dass wir nicht anders können, als das in Daten Gezeigte als etwas Pädagogisches zu sehen, sei es nun ein Lernen oder Leistung, obgleich wir wissen, dass sich dieses der eigentlichen Wahrnehmung entzieht. 


\subsection{Datenhandeln ist Waren austauschen}

Das letzte metaphorische Konzept ist als Datenhandeln ist Waren austauschen benannt, welches die Beteiligten gleichsam als Händlerinnen und Händler oder Handwerkerinnen und Handwerker kennzeichnet. Im Zusammenhang mit Datenhandeln werden hier vor allem Metaphoriken des Greifens, des Austauschs und des Bewegens von Daten verwendet. Daten werden herausgegeben, erfasst und Personen haben Zugriff auf sie. Daten werden somit als verstofflichte, bewegliche und begreifbare Objekte verstanden und sind sinnlich wahrnehmbar. Von Daten wird u. a. als in Behältern, wie Software oder Schulakte, befindlich gesprochen. Die Verstofflichung der Daten zeigt sich im weiteren Datenhandeln metaphorisch auf zwei verschiedene aber zusammenhängende Weisen: Zum einen ist Datenhandeln ein Austausch, bei dem u. a. verschickt und empfangen wird; zum anderen umfasst dies Handlungen, welche zwischen Personen, Systemen oder Organisationen vollzogen werden, wie die folgenden Zitate darlegen:

«Dann habe ich aber auch ähm irgendwie äh e cloudbasiertes System, wo ich sag, ich kann mit Schülerinnen und Schülern Lernmaterialien austauschen.» (SL2, Pos. 16, Herv. Autoren)

«Aber diese Daten überall einzupflegen und von einem System ins andere zu übertragen, das ist viel Arbeit und erfordert große Sorgfalt.» (SL4, Pos. 12, Herv. Autoren)

«Von der allgemeinbildenden Schule an die Grundschule werden keine Daten weitergegeben.»(SL1, Pos. 3, Herv. Autoren)

Allen drei Zitaten gemein ist das Betrachten von Daten als Objekte, die ausgetauscht, übertragen und weitergegeben werden. Die Unterscheidung zwischen Austausch und $\mathrm{Handel}$ ist in Abgrenzung zu anderen metaphorischen Konzepten wie «Geschäft» (Schmitt 2017, 230) zu treffen, denn für den Handel oder das Geschäft bedarf es einer Gewinnabsicht. Metaphorisch deutet nichts darauf hin, dass der Unterricht oder die Verwaltung durch Daten und Datenhandeln profitieren könnten, wertvoller wären oder sich die Arbeit mit Daten auszahlen könnte. Daher deutet das metaphorische Konzept des Warenaustausches darauf hin, dass ein Teil des Datenhandelns in Schule auf Zusammenarbeit ausgerichtet ist. Durch den Begriff Lernmaterialien, der auf Daten als Roh- oder Werkstoffe hinweist, lässt sich schlussfolgern, dass Daten nicht nur Objekte, sondern Waren sind. Dementsprechend werden Daten verwertet, genutzt und man macht etwas mit ihnen; eine mit Absicht vage, dennoch ökonomische Formulierung, denn anders als im direkt folgenden Zitat bleibt das Ergebnis des Machens oft unbestimmt. 
«[...] also die Statistik muss man ja machen. Also es gibt ja verbindliche Sachen, die wir dann weitergeben müssen.» (SL4, Pos. 7, Herv. Autoren)

«[...] dann überprüfen können wie oft Schüler oft diese Website eingeloggt waren welche Lektionen sie gelesen haben, ansonsten habe ich [...] niemanden gefunden der Daten verwertet, die von solchen Situationen anfallen.» (SL3, Pos. 4, Herv. Autoren)

«Also wir selbst nutzen diese Daten nicht. Das ist so ein Riesenaufwand, diese Daten zunächst mal zu erzeugen, damit die alle auch stimmig sind.» (SL1, Pos. 54, Herv. Autoren)

Die Metaphern des Datenhandelns sind darauf gerichtet, vorhandene Daten zu formen oder zu veredeln, ein Vorgang, der Arbeit und Fähigkeiten erfordert; damit sind die Handelnden (auch) handwerklich tätig. Wie genau Daten geformt oder veredelt werden oder welche Methoden Anwendung finden, obliegt den Handelnden. Inhaltlich wird aus den obigen Zitaten deutlich, dass es neben verpflichtendem ("Statistik muss man ja machen») auch optionales Handeln (Verwertung der LoginDaten) mit Daten gibt, was Schule die Möglichkeit einer Sinnzuschreibung an Daten und deren Produkt eröffnet. Die bereits angesprochene Unbestimmtheit der Ziele einer Arbeit mit Daten wird dadurch verstärkt, dass sich keine Metaphern sättigen liessen, welche beispielsweise die Optimierung von Verwaltungsabläufen oder die Anreicherung des Unterrichts enthalten. Datenhandeln erscheint hier fast als Selbstzweck.

\section{Diskussion der Ergebnisse}

Die rekonstruierten metaphorischen Konzepte, inklusive der verwendeten Metaphern im Sprechen der Schulleitungen, speisen sich aus unterschiedlichen Bereichen: der Ökonomie und Verwaltung, der Erkenntnis und in Teilen der Pädagogik sowie der Erziehung in Form von Pflege und Schützen. Datenhandeln gestaltet sich im Rahmen dieser Konzepte als zweckgerichtet, seien es die Be- respektive Verarbeitung von Daten, das Sichtbarmachen von Erkenntnissen, ein sensibler Umgang oder bekannte Verwaltungsakte.

Auffallend ist jedoch, dass der Ausgangspunkt (beispielsweise eine mögliche Gefahr, vor der es zu schützen gilt) oder das Ziel der jeweiligen Handlung in den meisten Fällen unterbestimmt bleiben - Datenhandeln wird damit fast zu einer selbstreferentiellen Tätigkeit an Schule. Weiterhin ist allen vier Konzepten gemeinsam, dass Daten in diesen eine passive Rolle einnehmen und vergegenständlicht werden. Dies wird auch durch ein wiederkehrendes Behälter- bzw. Raum-Schema untermalt, das 
die Passivität der Daten in ihrer Eingeschlossenheit impliziert (Schmitt 2017): Daten werden in diesem Kontext ergänzend dem Motiv des Tauschens (siehe Kapitel 3.2.) metaphorisch innerhalb verschiedener Räume respektive Level und Behälter platziert bzw. bewegt: Sie werden herein- und herausgelegt, darauf oder dahinter. Zusätzlich sind einige metaphorische Wendungen des Wassers (beispielsweise fliessen, Flut) im Material auszumachen, aber nicht zu sättigen. Daher wäre in weiteren Untersuchungen zu erörtern, ob Daten und Datenhandeln - auch wenn diese im vorliegenden Material nicht gesättigt werden konnten - in Konzepten von Mobilität verhandelt werden oder ob Datenhandeln durch Raum-Schemata strukturiert wird.

Ebenso kann von einer Überlappung der vier Konzepte ausgegangen werden. Datengestützte Verwaltungstätigkeiten kommen nicht ohne eine Form des Datenbearbeitens und des Datenschützens aus. Hierdurch offenbart sich gleichzeitig ein inhärentes Spannungsverhältnis: Denn Datenhandeln als Schützen und Pflegen sowie Tauschen und Veredeln versetzt pädagogisches Personal in die Dilemma-Situation, dieselben Daten zu bearbeiten und zu tauschen, die es zugleich zu schützen gilt.

Zieht man weitere metaphorische Konzepte hinzu, welche in Diskursen der Pädagogik auffindbar sind, so zeigt sich, dass die vier rekonstruierten Konzepte zu Datenhandeln daran Anschlussmöglichkeiten bieten respektive sich ähnliche Konzepte im schulischen Kontext finden lassen. So wird nach Schmitt (2017) Lernen u. a. mittels Metaphoriken des Sehens, Hegen und Pflegens, Weitergebens, des Geschäfts sowie Produzieren und Schaffens (vgl. ebd., 230) verhandelt. Betrachtet man somit beispielsweise das im Rahmen des Artikels vorgestellte Konzept des Schützens unter Perspektive des Lernens als Hegen und Pflegen, wirft dies Fragen auf, inwiefern hier eine Übertragung des Bildes von Schülerinnen und Schülern, welche im Garten "Schule» der Pflege bedürfen, auf Daten stattfindet, ob Daten lediglich anstelle dieser Betrachtung erfahren, Daten der Schülerinnen und Schüler gleichwertig gepflegt werden, wie die Personen von denen sie stammen, oder Schülerinnen und Schülern und ihre Daten gar gleichgesetzt werden.

Ähnliche Überlegungen zeigen, um ein zweites Beispiel anzuführen, der Vergleich des Lernens als Geschäft bzw. die Metapher der Materialbearbeitung (Guski 2007, 169) mit dem rekonstruierten Konzept des Warenaustauschs auf: Bedürfen Daten einer Veredelung bzw. Formung, spricht auch Guski von einer Bearbeitung und Formung durch Lehrpersonen. ${ }^{11}$ Demnach wirft auch diese gemeinsame Betrachtung Fragen nach der (kongruenten) Rolle von Daten und Schülerinnen und Schülern auf. Dieses Verhältnis gilt es, in folgenden Untersuchungen näher zu betrachten.

Ein weiterer Ausgangspunkt für zukünftige Untersuchungen bietet die Erweiterung des Zielbereichs der Metaphernanalyse. So liessen sich interviewübergreifend metaphorische Wendungen identifizieren, welche die schulische Infrastruktur als Fundament (Metaphorik des Hauses) für Digitalisierung und damit auch implizit

11 Allerdings hier von Schülerinnen und Schülern als Material. 
Datenhandeln verbildlichen. Auffallend gestaltete sich, insbesondere in diesem Kontext, dass die befragten Schulleitungen in ihren Ausführungen nicht immer eindeutig zwischen Digitalisierung, dem Einsatz digitaler Medien und Daten unterschieden, wodurch sich auch hier Forschungsimplikationen zum Verhältnis und dem Verständnis von Digitalisierung und Daten des Schulleitungspersonals ergeben.

\section{Limitation und Ausblick}

Abschliessend sei erneut darauf verwiesen, dass die zugrundeliegenden Interviews mit Schulleitungen geführt wurden, weshalb die rekonstruierten Konzepte eine starke Prägung aus der Leitungsperspektive aufweisen und sich Metaphern des Verwaltens allein aufgrund der Tätigkeit aufdrängen. Dies lässt sich anhand zweier Aspekte besonders verdeutlichen: Einerseits liessen sich metaphorische Wendungen des datengestützten Gestaltens oder Konstruierens (Metaphorik des Hauses), welche beispielsweise in der Verbindung von Datenhandeln und Unterrichtsgestaltung zu erwarten wären, nicht sättigen. Andererseits beschränkt sich auch die metaphorische und inhaltliche Thematisierung der Datenherkunft auf Bereiche des Verwaltens, Personenbezugs und der Benotung. Nichtsdestoweniger bieten die hier identifizierten Bereiche erste Implikationen, die im Rahmen des Forschungsprojekts als Ausgangspunkte für eine weitere Untersuchung pädagogischen Handelns im schulischen Kontext dienen. Eine Rekonstruktion metaphorischer Konzepte von Lehrkräften ohne Verwaltungs- oder Leitungsaufgaben sowie weiterem pädagogischen Personal soll daher im Forschungsprojekt forciert werden, um einen Vergleich und eine Perspektivenerweiterung zu ermöglichen.

\section{Literatur}

Ackeren, Isabell van, Klaus Klemm, und Svenja Mareike Kühn. 2015. Entstehung, Struktur und Steuerung des deutschen Schulsystems: eine Einführung. 3. Aufl. Wiesbaden: VS Verlag für Sozialwissenschaften. https://doi.org/10.1007/978-3-531-20000-2.

Ackeren, Isabell van. 2003. Evaluation, Rückmeldung und Schulentwicklung: Erfahrungen mit zentralen Tests, Prüfungen und Inspektionen in England, Frankreich und den Niederlanden. Bd. 2. Studien zur international und interkulturell vergleichenden Erziehungswissenschaft. Münster; München; Berlin [u.a.]: Waxmann.

Altrichter, Herbert, und Katharina Maag Merki, Hrsg. 2010. Handbuch Neue Steuerung im Schulsystem. 1. Aufl. Bd. 7. Educational governance. Wiesbaden: VS Verlag für Sozialwissenschaften. https://doi.org/10.1007/978-3-531-92245-4. 
Breiter, Andreas, und Andreas Hepp. 2018. «The Complexity of Datafication: Putting Digital Traces in Context». In Communicative Figurations. Transforming Communications - Studies in Cross-Media Research, herausgegeben von Andreas Hepp, Andreas Breiter, und Uwe Hasebrink. Palgrave Macmillan, Cham. https://doi.org/10.1007/978-3-319-65584-0_19.

Breiter, Andreas, und Juliane Jarke. 2019. «Editorial: the datafication of education. Learning». Media and Technology 44(1):1-6. https://doi.org/10.1080/17439884.2019.1573833.

Dalton, Craig M., und Jim Thatcher. 2014. Inflated Granularity: The Promise of Big Data and the Need for a Critical Data Studies. Presentation at the Annual Meeting of the Association of American Geographers, Tampa, Fl, April 9, 2014.

Dander, Valentin. 2015. «Diskurse + Praktiken = Datenhandeln?» Medienimpulse 53(3). https:// doi.org/10.21243/mi-03-15-02.

Dander, Valentin, und Sandra Aßmann. 2015. «Medienpädagogik und (Big) Data: Konsequenzen für die erziehungswissenschaftliche Medienforschung und -praxis.» In Big Data und Medienbildung. Schriftenreihe zur digitalen Gesellschaft NRW, herausgegeben von Harald Gapski, 33-50. Düsseldorf; München: kopaed. http://nbn-resolving.org/urn:nbn:de:0111pedocs-116340.

Forell, Matthias, Gabriele Bellenberg und Grit im Brahm. 2019. «Das Gymnasium im Zuge fortschreitender Öffnung». In Bildungsforschung mit Daten der amtlichen Statistik, Bd. 14, Die Deutsche Schule, herausgegeben von Detlef Fickermann, und Horst Weishaupt, 215-233. Münster; New York: Waxmann.

Fuller, Matthew. 2008. Software Studies / a lexicon. Cambridge, MA: MIT Press.

Gehring, Petra. 2010. «Erkenntnis durch Metaphern? Methodologische Bemerkungen zur Metaphernforschung». In Metaphern in Wissenskulturen, herausgegeben von Matthias Junge, 203-220. Wiesbaden: VS Verlag für Sozialwissenschaften. https://doi.org/10.1007/978-3531-92164-8_12.

Gießmann, Sebastian, und Marcus Burkhardt. 2014. «Was ist Datenkritik? Zur Einführung». http://www.medialekontrolle.de/wp-content/uploads/2014/09/Giessmann-SebastianBurkhardt-Marcus-2014-03-01.pdf.

Grek, Sotiria. 2009. «Governing by Numbers: The PISA 'Effect' in Europe.» Journal of Education Policy 24(1): 23-37. https://doi.org/10.1080/02680930802412669.

Guski, Alexandra. 2007. Metaphern der Pädagogik: metaphorische Konzepte von Schule, schulischem Lernen und Lehren in pädagogischen Texten von Comenius bis zur Gegenwart. Bd. 53. Explorationen. Bern; Berlin; Frankfurt am Main; Wien [u.a.]: Lang.

Hartong, Sigrid. 2016. «Between Assessments, Digital Technologies and Big Data: The Growing Influence of 'Hidden' Data Mediators in Education». European Educational Research Journal 15 (5) (September 2016): 523-36. https://doi.org/10.1177/1474904116648966.

Herzog, Walter. 1994. «Pädagogische Metaphern und ihre körperlichen Wurzeln.» Referat vom 17. Juni 1994 am Pestalozzianum Zürich. Abgerufen am 13.01.2020. https:// www.walterherzog.ch/app/download/12325254523/Pa\%CC\%88dagogische+Metaphern. pdf?t=1502117178. 
Houben, Daniel, und Biance Prietl, Hrsg. 2018. Datengesellschaft. Einsichten in die Datafizierung des Sozialen. Bielefeld: transcript. https://doi.org/10.14361/9783839439579.

Ifenthaler, Dirk, und Clara Schumacher. 2016. «Learning Analytics im Hochschulkontext». WiSt - Wirtschaftswissenschaftliches Studium 45 (4): 176-81. https://doi.org/10.15358/03401650-2016-4-176.

Kade, Jochen, und Wolfgang Seitter. 2007. «Offensichtlich unsichtbar. Die Pädagogisierung des Umgangs mit Wissen im Kontext des lebenslangen Lernens». Zeitschrift für Erziehungswissenschaft 10(2) 2: 181-98. https://doi.org/10.1007/s11618-007-0026-7.

Kitchin, Rob, und Tracey P. Lauriault. 2014. «Towards Critical Data Studies: Charting and Unpacking Data Assemblages and Their Work (July 30, 2014)». The Programmable City Working Paper 2; preprint version of chapter to be published in Geoweb and Big Data, edited by Josef Eckert, Andrew Shears, and Jim Thatcher. University of Nebraska. Press. Forthcoming. Available at SSRN: https://ssrn.com/abstract=2474112.

Koselleck, Reinhart. 2006. Begriffsgeschichten. Studien zur Semantik und Pragmatik der politischen und sozialen Sprache. Frankfurt a.M.: Suhrkamp.

Krieter, Philipp. 2020. Looking Inside - Mobile Screen Recordings as a Privacy Friendly Long-Term Data Source to Analyze User Behavior. Bremen. https://doi.org/10.26092/elib/103.

Krieter, Philipp, und Andreas Breiter. 2020. «Digitale Spuren von Studierenden in virtuellen Lernumgebungen». In Studierende - Medien - Universität. Einblicke in studentische Medienwelten, herausgegeben von Sandra Hofhues, Mandy Schiefner-Rohs, Sandra Aßmann, und Taiga Brahm, 131-52. Münster: Waxmann. https://doi.org/10.31244/9783830990499.

Lachney, Michael, William Babbitt, und Ron Eglash. 2016. "Software Design in the «Construction Genre of Learning Technology: Content Aware versus Content Agnostic». Computational Culture. http://computationalculture.net/software-design-in-the-construction-genreof-learning-technology-content-aware-versus-content-agnostic/.

Lakoff, George, und Mark Johnson. 1980. Metaphors we live by. Chicago: The University of Chicago Press.

Mau, Steffen. 2017. Das metrische Wir: über die Quantifizierung des Sozialen. Frankfurt/Main.

Pöggeler, Franz, Hrsg. 1992. Bild und Bildung. Frankfurt a.M.: Peter Lang Verlag.

Saban, Ahmet. 2004. «Prospective classroom teachers' metaphorical images of selves and comparing them to those they have of their elementary and cooperating teachers». International Journal of Educational Development 24: 617-35. https://doi.org/10.1016/j.ijedudev.2004.03.003.

Schiefner, Mandy, und Balthasar Eugster. 2010. «Sichtbarkeit von Lehre erhöhen - Gedanken am Beispiel des Lehrpreises». In ‘Ausgezeichnete Lehre!〉 Lehrpreise an Universitäten. Erörterungen - Konzepte - Vergabepraxis, herausgegeben von Peter Tremp, 71-88. Münster: Waxmann.

Schmitt, Rudolf. 2017. Systematische Metaphernanalyse als Methode der qualitativen Sozialforschung. Wiesbaden: Springer VS. https://doi.org/10.1007/978-3-658-13464-8. 
Schmitt, Rudolf, Julia Schröder, und Larissa Pfaller. 2018. Systematische Metaphernanalyse: Eine Einführung. Wiesbaden: Springer VS. https://doi.org/10.1007/978-3-658-21460-9.

Seiler, Luisa, Matthias Kuhnel, Andrea Honal, und Dirk Ifenthaler. 2018. «Mobile Learning Analytics: Potenziale für Lernen und Lehren am Beispiel Hochschule». In Handbuch Mobile Learning, herausgegeben von Claudia de Witt und Christina Gloerfeld, 585-612. Wiesbaden: Springer Fachmedien Wiesbaden. https://doi.org/10.1007/978-3-658-19123-8.

Selwyn, Neil. 2020. «Just playing around with Excel and pivot tables - the realities of datadriven schooling». Research Papers in Education. https://doi.org/10.1080/02671522.2020. 1812107.

Treml, Alfred K. 2000. Allgemeine Pädagogik: Grundlagen, Handlungsfelder und Perspektiven der Erziehung. Bd. 441. Kohlhammer-Urban-Taschenbücher. Stuttgart; Berlin; Köln: Kohlhammer.

Yeats, W. B. (1949). The Poems. London: MacMillan. 